\title{
Modular Zwitterion-Functionalized Poly(isopropyl methacrylate) Polymers for Hosting Luminescent Lead-Halide Perovskite Nanocrystals
}

\author{
Theodore A. Cohen, ${ }^{\dagger, \mathrm{a}}$ Y unping Huang, ${ }^{\dagger, \mathrm{b}}$ Nico A. Bricker, ${ }^{\mathrm{c}}$ Connor S. Juhl, ${ }^{\mathrm{c}}$ Tyler J. Milstein, ${ }^{\mathrm{c}}$ \\ J. Devin Mackenzie, ${ }^{\text {b,d }}$ Christine K. Luscombe, ${ }^{*, a, b, c}$ Daniel R. Gamelin*,a,c \\ ${ }^{a}$ Molecular Engineering and Sciences Institute, University of Washington, Seattle, Washington, \\ 98195, United States \\ ${ }^{b}$ Department of Materials Science and Engineering, University of Washington, \\ Seattle, Washington, 98195, United States \\ ${ }^{c}$ Department of Chemistry, University of Washington, Seattle, Washington, 98195-1700, United \\ States \\ ${ }^{d}$ Department of Mechanical Engineering, University of Washington, Seattle, Washington 98195, \\ United States \\ *Email: gamelin@uw.edu,luscombe@uw.edu \\ ${ }^{\dagger}$ These authors contributed equally to this work.
}

\section{Experimental Procedures}

Physical measurements. Nanocrystal (NC) transmission electron microscopy (TEM) images were collected using a FEI TECNAI F20 microscope operated at $200 \mathrm{kV}$. TEM samples were prepared by drop casting NC suspensions onto carbon-coated copper grids obtained from TED Pella, Inc. Edge length distribution data were collected with ImageJ and uncertainties associated with TEM size distributions are reported as standard deviations. Powder X-ray diffraction (XRD) data were collected using a Bruker D8 Discover diffractometer. Samples were prepared by drop-casting NC suspensions onto monocrystalline silicon wafer substrates. Samples were then irradiated using a high-efficiency $\mathrm{I} \mu \mathrm{S}$ microfocus X-ray source for $\mathrm{Cu} \mathrm{K} \alpha$ radiation $(50 \mathrm{kV}, 1 \mathrm{~mA})$ to obtain the reported data. Crystal structures were identified using reference patterns from the Inorganic Crystal Structure Database. Samples for ${ }^{1} \mathrm{H}$ nuclear magnetic resonance (NMR) measurements were prepared by dissolving zwitterionic polymers in $\sim 0.5 \mathrm{~mL}$ acetone- $\mathrm{d}_{6}$ or methanol- $\mathrm{d}_{4}$. For the NC/ZP3 NMR sample, a NC/polymer composite was prepared according to the hexanes precipitation procedure described in the main text. Once prepared, the sample was dried under vacuum to remove residual solvent and was dispersed in acetone- $\mathrm{d}_{6}$. Spectra were collected with an AV-300 NMR system operating at a ${ }^{1} \mathrm{H}$ frequency of 300.13 MHz. Raw data from the spectrometer were processed with the SpinWorks v4.2.8 software.

Spectroscopic measurements. Absorption spectra of solutions and solid samples were collected at room temperature using Cary 60 and Cary 500 spectrometers, respectively. Photoluminescence (PL) spectra were collected using a homebuilt setup with $365 \mathrm{~nm}$ diode excitation and an Ocean Optics 2000+ USB photodetector. Spectra were calibrated with an Ocean Optics deuterium-tungsten halogen light source. Time-resolved PL (TRPL) measurements 
were collected at room temperature using an Edinburgh FLS1000 spectrometer and pulsed excitation was provided by a $405 \mathrm{~nm}$ Edinburgh picosecond laser. Emission from the samples was collected through a monochromator-coupled PMT detector. PLQY measurements in the visible regime were performed with a Hamamatsu integrating sphere quantum-yield measurement system with $450-\mathrm{nm}$ excitation. Solution-state PLQYs were collected at various concentrations due to the dependence of PLQY on ligand concentration. ${ }^{1}$ These concentrations are summarized by an absorption percentage, which is calculated by comparing the integrated excitation light intensity for a given $\mathrm{NC}$ concentration with the integrated excitation light intensity for a blank cuvette filled with solvent. For as-synthesized NCs and solvated NC/ZFP3 samples, the main text reports PLQYs of the highest and lowest concentration samples, respectively. $\mathrm{Yb}^{3+} \mathrm{PL}$ was collected through an integrating sphere using a monochromator coupled to a spectrally corrected nitrogen-cooled CCD. Relative PL calibration was performed according to procedures described previously. ${ }^{2}$ PL microscope images were collected with a Nikon Eclipse LV150N digital microscope. A $450 \mathrm{~nm}$ excitation source was focused onto the sample with a 50x objective lens and the resultant PL was imaged with a CCD camera.

PL Lifetime Analysis. Raw PL decay curves were first normalized at their maxima and were then fit to the following model: $:^{3-4}$

$$
I(t)=\sum_{i=0}^{n} A_{i} e^{-\frac{t}{\tau_{i}}}
$$

where $I(t)$ is the number of photon counts as a function of pulse delay time, $A_{i}$ is the amplitude of a decay component with a lifetime of $\tau_{i}$, and $n$ is the number of exponents used in the fit. The data were fit using a custom Python scrip employing the SciPy software package. Once fit, the average weighted lifetime was calculated using the following equation: ${ }^{5-6}$

$$
\tau_{\text {avg }}=\frac{\sum_{i=0}^{n} A_{i} \tau_{i}{ }^{2}}{\sum_{i=0}^{n} A_{i} \tau_{i}}
$$

Stability Under Illumination. NC stabilities under illumination were measured using a Chanzon, $50 \mathrm{~W}, 450 \mathrm{~nm}$ LED light source attached to a heat sink and cooled with a 12V DC fan. The LED warmed up for 15 min before use and was separated from the sample by a large shutter to ensure that the entire sample was uniformly illuminated. The LED power was adjusted to provide an irradiance of $\sim 90 \mathrm{~mW} \mathrm{~cm}{ }^{-2}$, which was measured with a Coherent energy meter through a $1 \mathrm{~mm}$ diameter pinhole. PL data for these stability measurements were collected using a fiber-coupled ASEQ instruments LR1-T spectrometer. The spectrometer was operated in continuous mode with spectra collected every $3 \mathrm{~s}$ for the first $20 \mathrm{~min}$ and every $5 \mathrm{~min}$ for the remainder of the experiment. Spectra were integrated from 525 to $550 \mathrm{~nm}$ and 600 to $800 \mathrm{~nm}$ for $\mathrm{CsPbBr}_{3}$ and $\mathrm{CsPbI}_{3} \mathrm{NCs}$, respectively, to determine $\mathrm{PL}$ intensity as a function of illumination time. 


\section{Supporting Figures.}

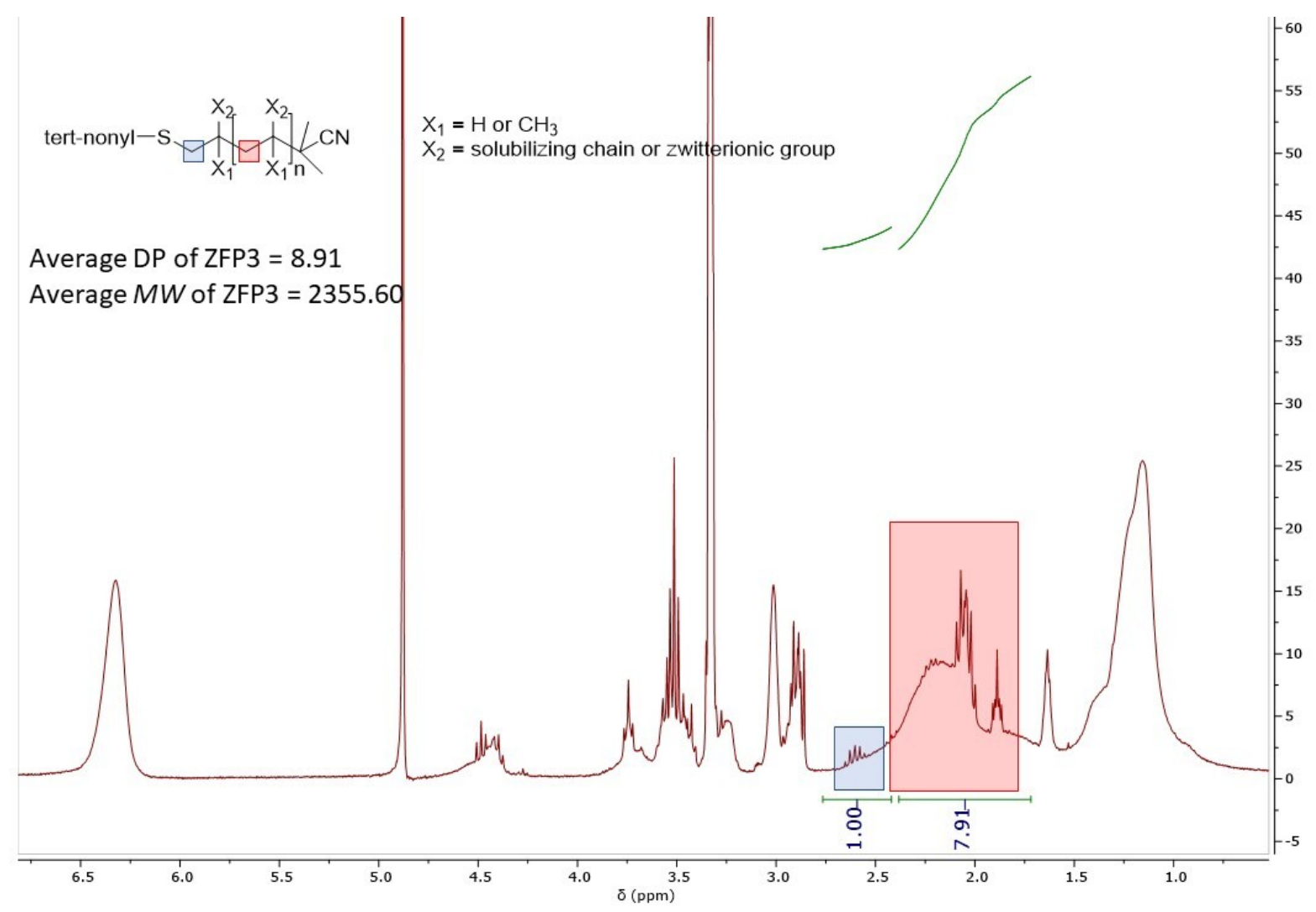

Figure S1. NMR spectrum of ZFP3 in methanol-d $\mathrm{d}_{4}$ measured at room temperature. The NMR peaks used to calculate degree of polymerization (DP) and molecular weight (MW) are highlighted. 


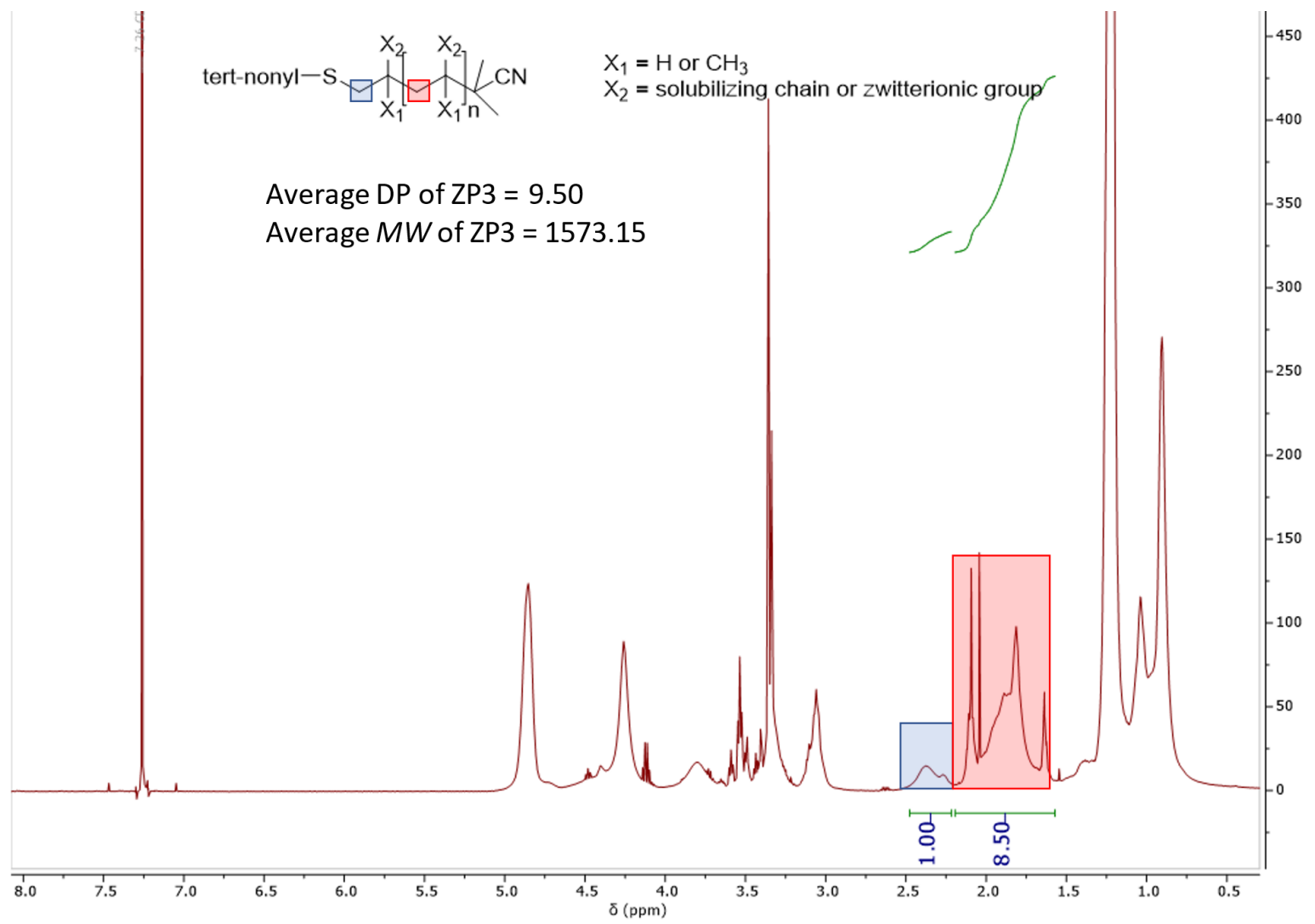

Figure S2. NMR spectrum of ZP3 in methanol- $\mathrm{d}_{4}$ measured at room temperature. The NMR peaks used to calculate DP and MW are highlighted. 


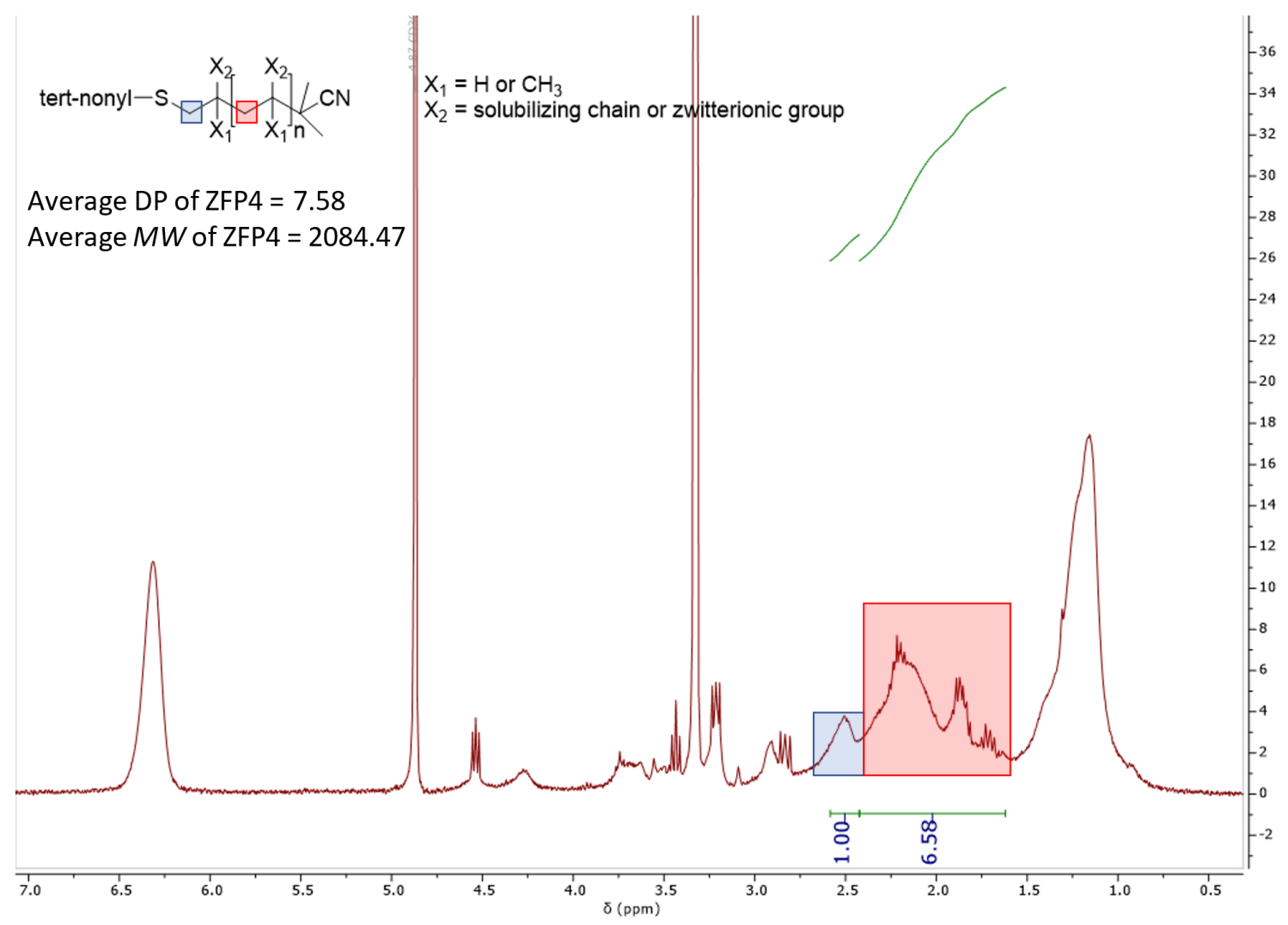

Figure S3. NMR spectrum of ZFP4 in methanol- $\mathrm{d}_{4}$ measured at room temperature. The NMR peaks used to calculate DP and MW are highlighted. This polymer was not used in this work because it was not soluble in ethyl or butyl acetate. 


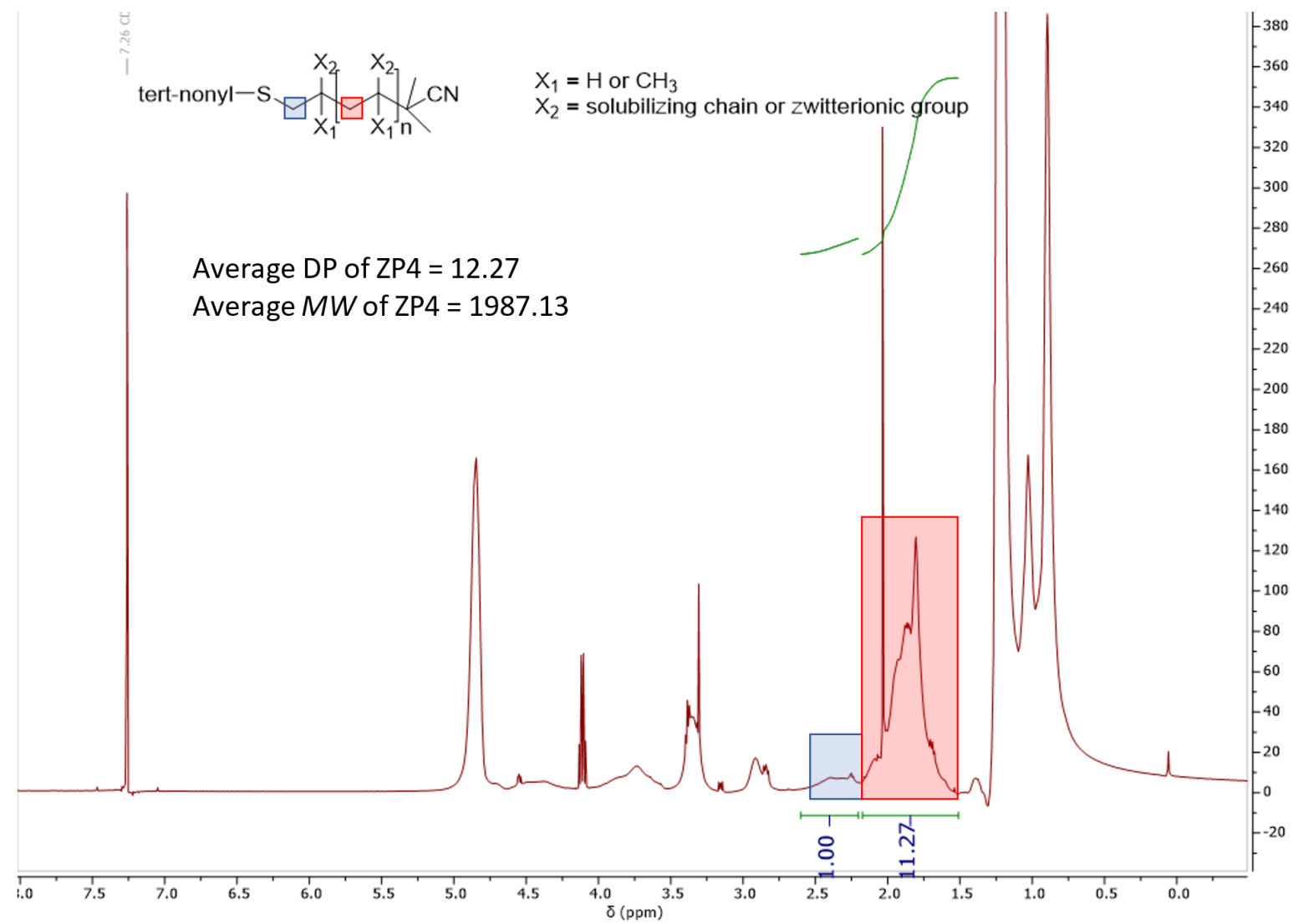

Figure S4. NMR spectrum of ZP4 in methanol- $\mathrm{d}_{4}$ measured at room temperature. The NMR peaks used to calculate DP and MW are highlighted.
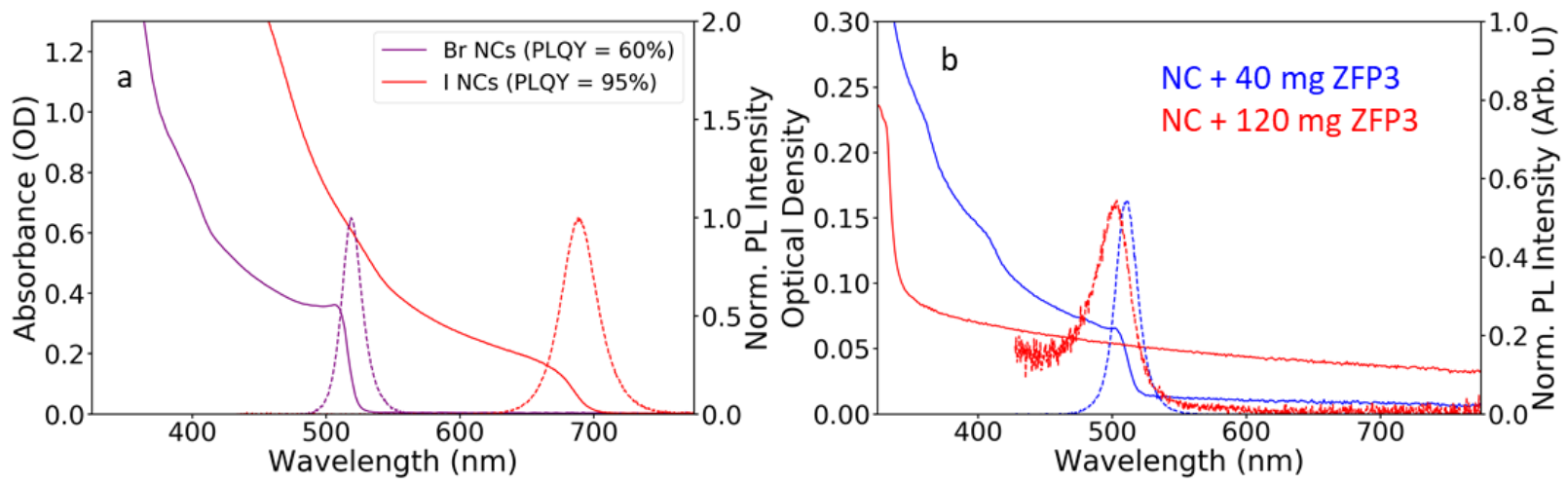

Figure S5. (a) Absorption and PL spectra of as-synthesized $\mathrm{CsPbBr}_{3}$ and $\mathrm{CsPbI}_{3} \mathrm{NCs}$. Solution-state PLQY values are provided in the legend. (b) Absorption spectra of $\mathrm{CsPbBr}_{3} \mathrm{NCs}$ in the hexane supernatant after purification of the NC/ZFP3 composite from ethyl acetate. When less ZFP3 is used, a small amount of NCs are present in the supernatant, and the majority of the NCs precipitate as a NC/ZFP3 composite. When more ZFP3 is used, almost all NCs are transferred into the composite. 


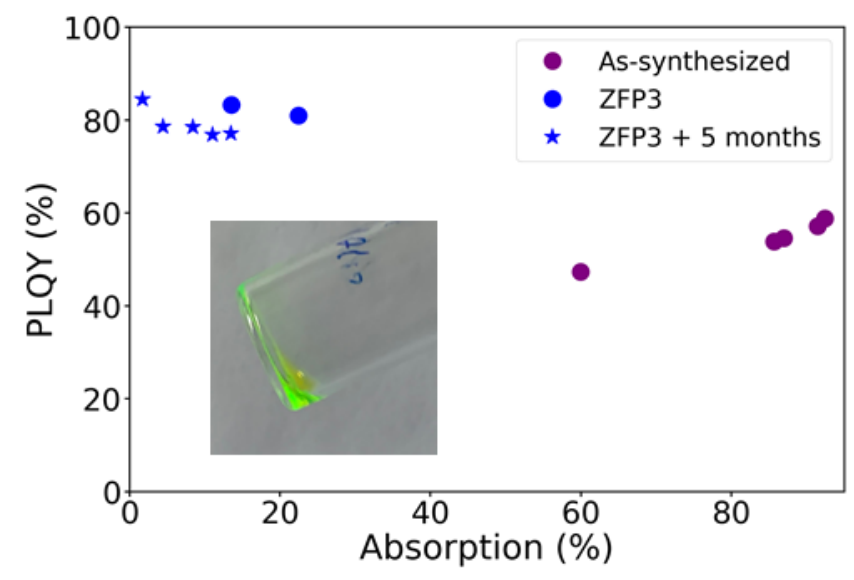

Figure S6. PLQY as a function of absorption percentage of as-synthesized $\mathrm{CsPbBr}_{3} \mathrm{NCs}$ in hexanes and after transfer into a ZFP3 composite in butyl acetate at room temperature. As-synthesized NC solutions needed to be stored in a $\mathrm{N}_{2}$-filled glovebox, while NC/ZFP3 solutions did not. Inset: Photograph of $\mathrm{CsPbBr}_{3} \mathrm{NC} / \mathrm{ZFP} 3$ solution after 5 months of dark, ambient storage.
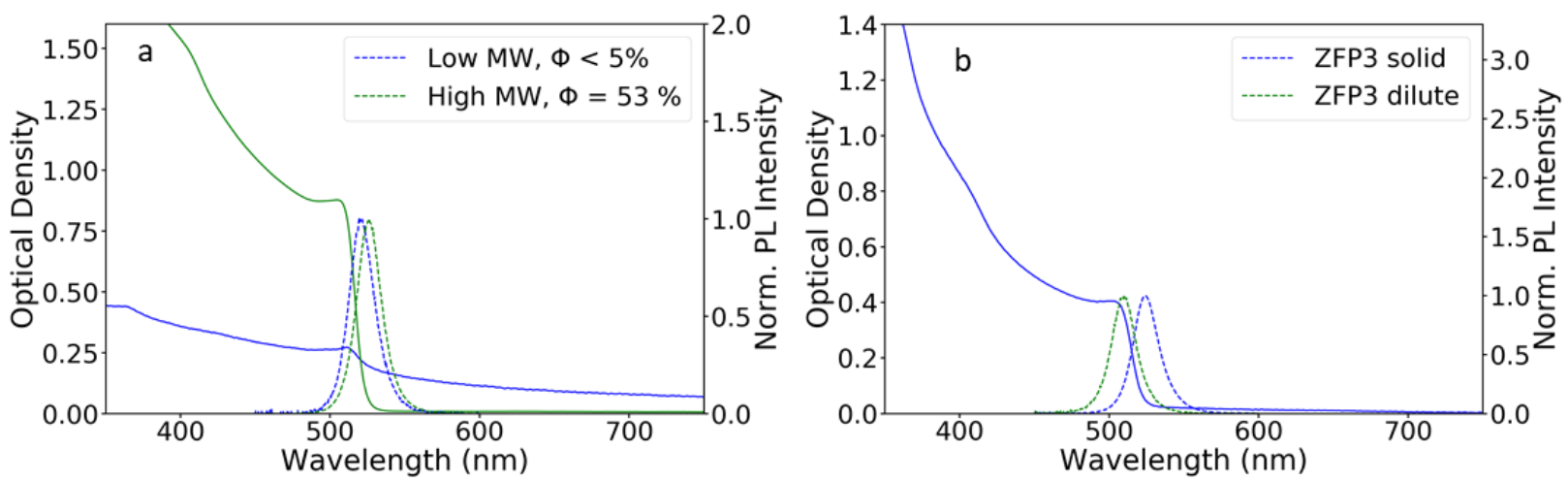

Figure S7. (a) Absorption and $\mathrm{PL}$ spectra of $\mathrm{CsPbBr}_{3}$ NC/PMMA composites. Aggregation was observed when the toluene evaporated from a drop-cast solution of NCs and low-molecular-weight (MW) PMMA, but aggregation was not observed when highMW PMMA was used. (b) Absorption spectrum of a representative high-concentration NC/ZFP3 composite and PL spectra of a representative NC/ZFP3 composite drop cast from high- and low-concentration solutions of butyl acetate. Reabsorption-induced redshifts were observed for all samples analyzed in this work. 

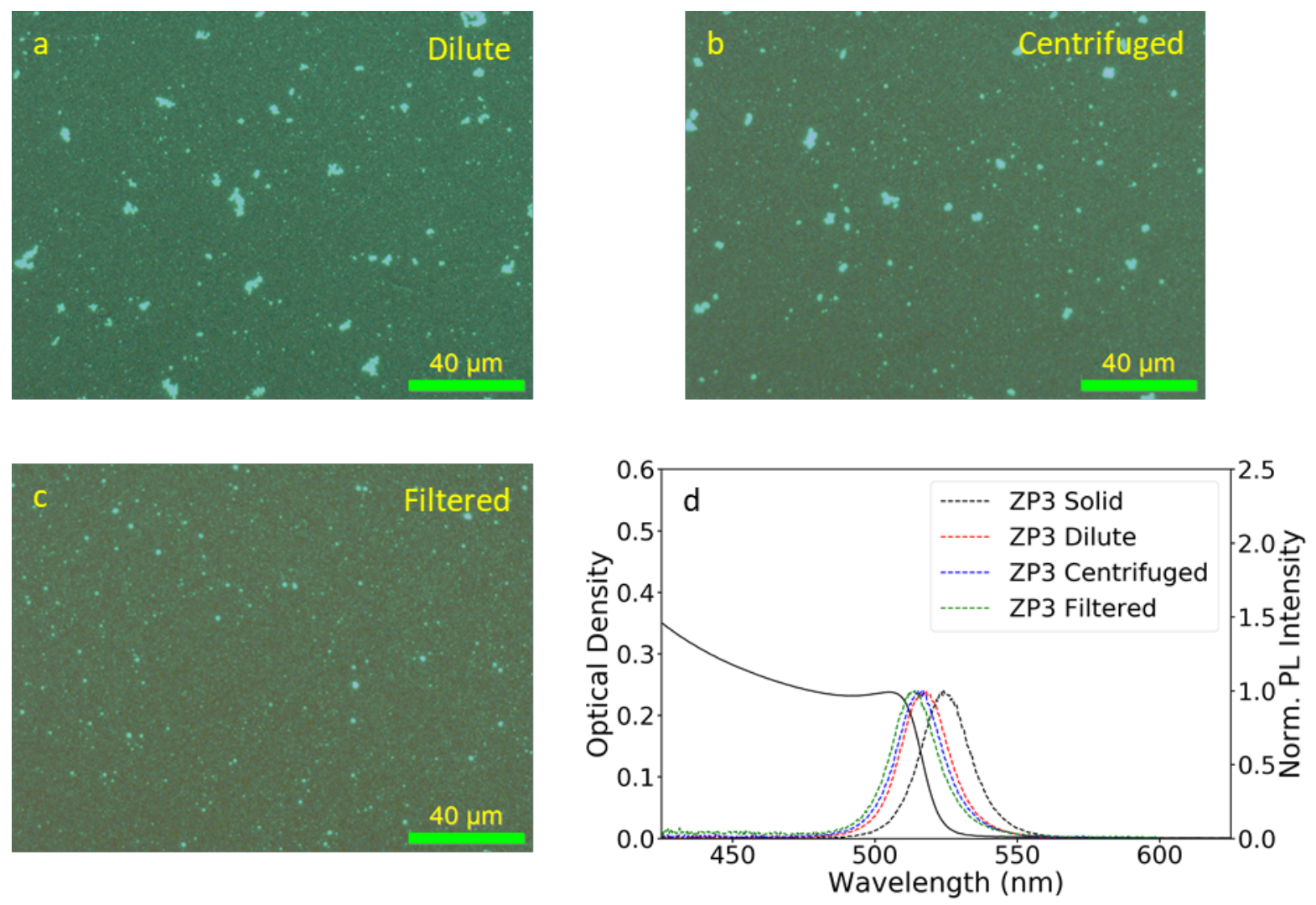

Figure S8. $\mathrm{PL}$ microscope images of representative solid-state $\mathrm{CsPbBr}_{3} \quad \mathrm{NC} / \mathrm{ZP} 3$ composites after (a) dilution of the original NC/ZP3 butyl acetate solution by a factor of 50 , (b) centrifugation of the diluted $\mathrm{NC} / \mathrm{ZP} 3$ solution, and (c) filtering of the centrifuged, diluted NC/ZP3 solution through a $0.22 \mu \mathrm{m}$ PTFE syringe filter prior to drop casting. The processes of centrifugation and filtering successfully removed large aggregates from these composites. (d) Absorption and PL spectra of a high-optical density $\mathrm{CsPbBr}_{3}$ NC/ZP3 composite film plotted with the PL spectra of the three low-optical density films shown in panels (a)-(c). The slight blue shift observed after centrifugation and filtration suggests that a small fraction of the PL redshifts observed in these samples can be attributed to aggregation, but reabsorption is the main contributor to the observed PL redshifts for high-optical density samples. 

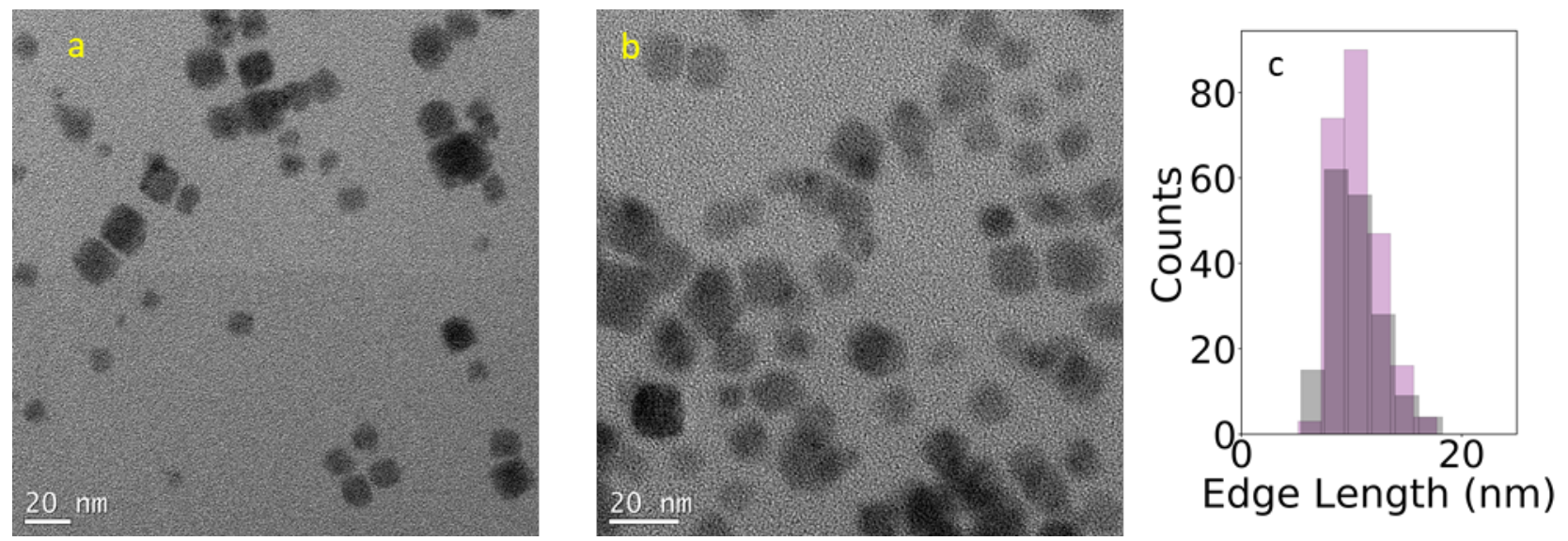

Figure S9. (a, b) Additional TEM images of $\mathrm{CsPbBr}_{3} \mathrm{NCs}$ drop cast from a butyl acetate solution of NC/ZP3 composite. These images were used to acquire additional data for analysis of particle size distributions. (c) Histogram of edge lengths from the TEM images shown in panels $a, b$ and Figure $1 \mathrm{c}, \mathrm{d}$ of the main text.
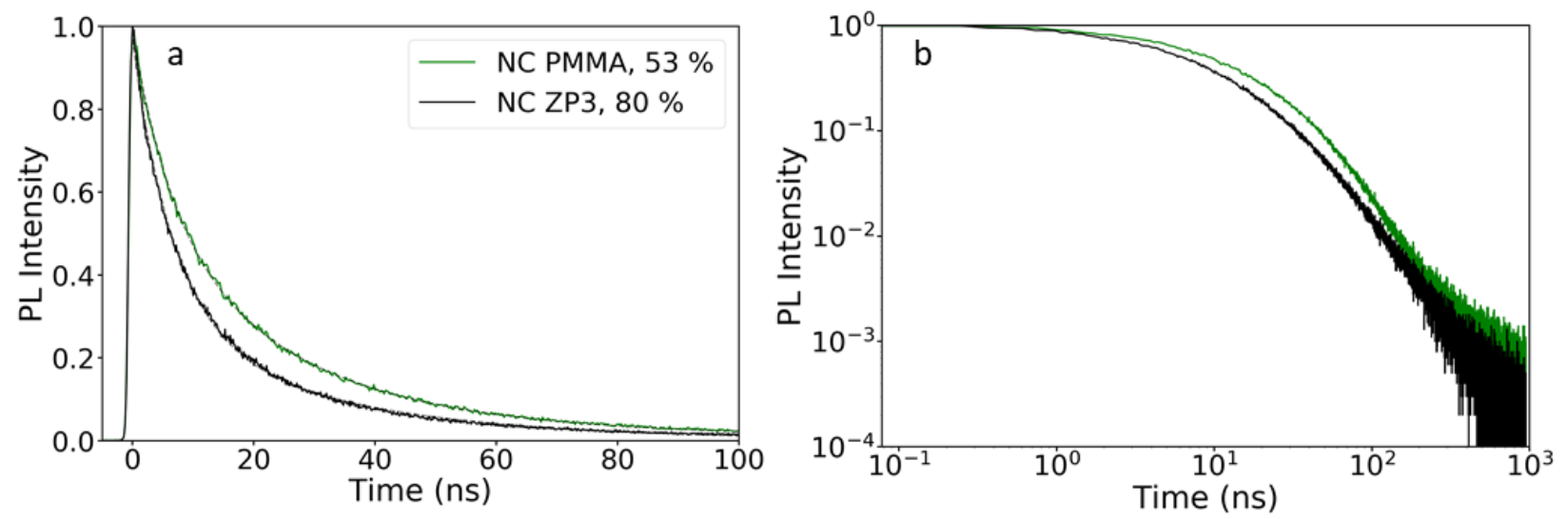

Figure S10. (a) PL decay curves measured in ambient conditions with $405 \mathrm{~nm}$ excitation for $\mathrm{CsPbBr}_{3} \mathrm{NCs}$ in a PMMA and a ZP3 composite, normalized at $t=0$. Gray dashed traces correspond to the result of biexponential and triexponential decay fits for NCs in ZP3 and PMMA composites, respectively. (b) Log - log plot of the PL decay data shown in panel (a). These data appear to follow a power-law relationship at long times after the excitation pulse, suggesting the presence of delayed exciton luminescence due to reversible trapping. ${ }^{4,6-9}$ 
Table S1. Fitting parameters from fits to the PL lifetime data shown in Figure S10a.

\begin{tabular}{|c|c|c|}
\hline parameter & PMMA & ZP3 \\
\hline$\tau_{1}$ & $6.3 \mathrm{~ns}$ & $6.8 \mathrm{~ns}$ \\
$\% \tau_{1}$ & $42.1 \%$ & $70.9 \%$ \\
$\tau_{2}$ & $23.7 \mathrm{~ns}$ & $31.1 \mathrm{~ns}$ \\
$\% \tau_{2}$ & $54.3 \%$ & $29.1 \%$ \\
$\tau_{3}$ & $111 \mathrm{~ns}$ & \\
$\% \tau_{3}$ & $3.5 \%$ & \\
$\tau_{\text {avg }}$ & $39.3 \mathrm{~ns}$ & $22.7 \mathrm{~ns}$ \\
\hline
\end{tabular}

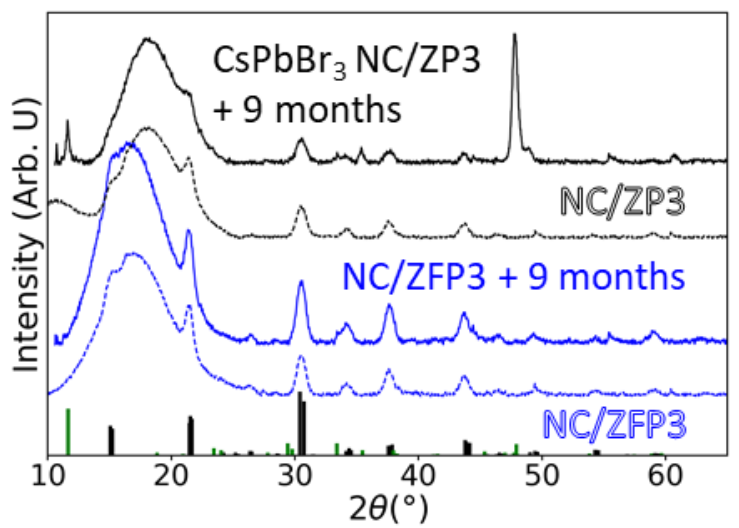

Figure S11. XRD data for $\mathrm{CsPbBr}_{3} \mathrm{NCs}$ in ZFP3 and ZP3 composites collected after preparation and after 9 months of dark storage. The additional peaks that appear in the NC/ZP3 data after 9 months index to the $\mathrm{CsPb}_{2} \mathrm{Br}_{5}$ crystal structure (PDF-254290, green bars) rather than the $\mathrm{CsPbBr}_{3}$ crystal structure (PDF-97851, black bars). Despite the appearance of $\mathrm{CsPb}_{2} \mathrm{Br}_{5}$, the PLQYs of the NCs in ZFP3 and ZP3 remained constant at 59 and $85 \%$, respectively after 1 year of dark storage. 

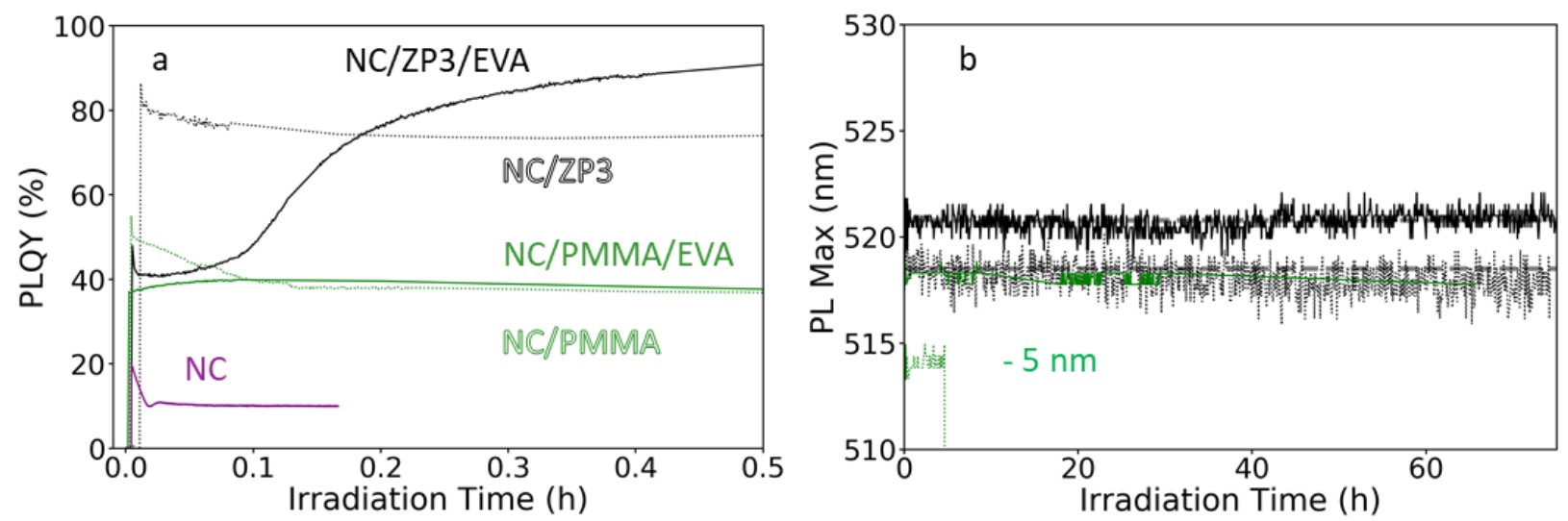

Figure S12. (a) PLQYs measured during illumination of the $\mathrm{CsPbBr}_{3} \mathrm{NC} / \mathrm{ZP} 3$ and NC/PMMA composites with and without EVA encapsulation from Figure $2 d$ of the main text and of unencapsulated, drop-cast films of NCs, plotted over the first half hour. Photobrightening is observed in encapsulated samples over the first 40 min of irradiation. (b) PL maximum as a function of illumination time for laminated and non-laminated samples from panel (a). There is very little shift in the PL maxima in all cases. The PL maximum for the NCs in PMMA without EVA encapsulation has been artificially shifted down by $5 \mathrm{~nm}$ for ease of viewing.

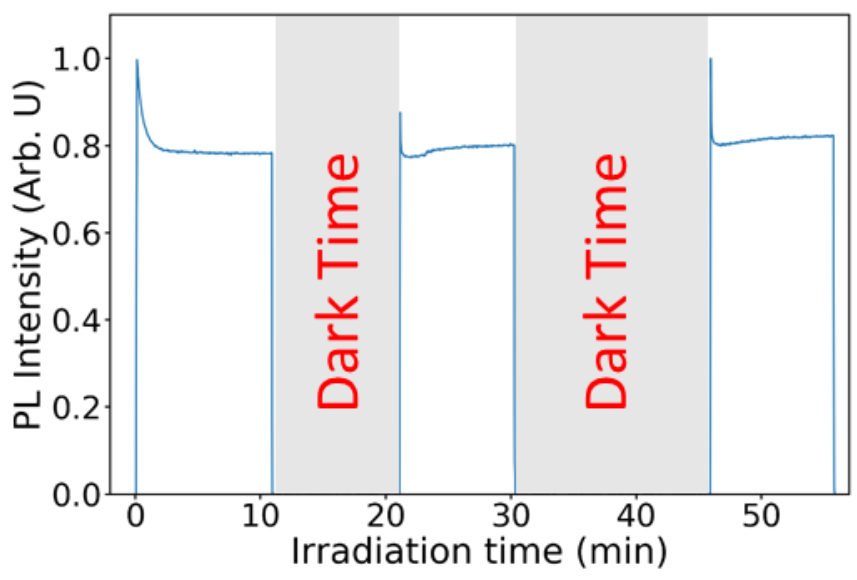

Figure S13. PL intensity of a $\mathrm{CsPbBr}_{3} \mathrm{NC} / \mathrm{ZP} 3$ composite plotted as a function of illumination time using $450 \mathrm{~nm}, \sim 90 \mathrm{~mW} \mathrm{~cm}^{-2}$ LED illumination. The LED shutter was periodically opened and closed to demonstrate that the PL intensities can fully recover after a short amount of dark recovery time but then decay again with continued illumination. 


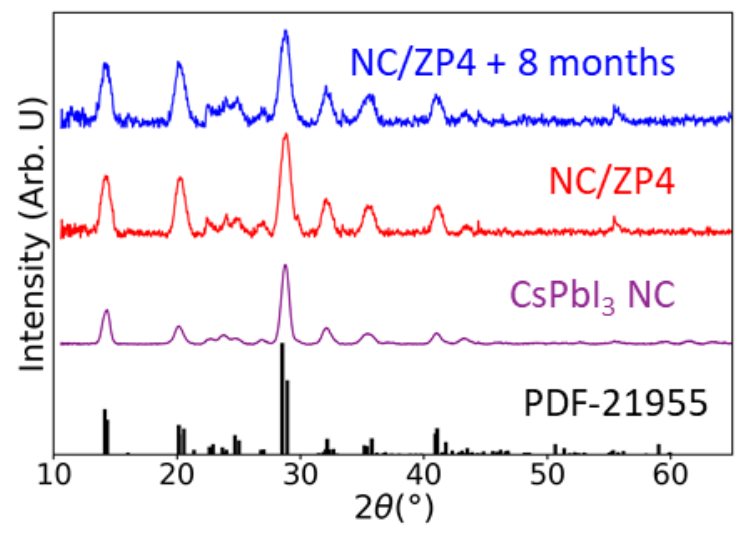

Figure S14. $\mathrm{XRD}$ data for $\mathrm{CsPbI}_{3} \mathrm{NCs}$ in their as-synthesized and NC/ZP4 composite forms. XRD data were collected for the same NC/ZP4 composite following preparation and again after 8 months of dark storage. Both measurements show peaks that are consistent with the $\gamma$-cubic $\mathrm{CsPbI}_{3}$ phase. The FWHM of the peak at $28^{\circ}$ increases by about $0.155^{\circ}$ (from $0.742^{\circ}$ to $0.897^{\circ}$ ) upon formation of the NC/ZP4 composite, suggesting that the nanocrystals may be slightly etched by the polymer transfer process. Neither the crystal structure nor the FWHM appears to change substantially after 8 months of dark storage. The PLQY of the NC/ZP4 composite was $60 \%$ after 1 year of dark storage.

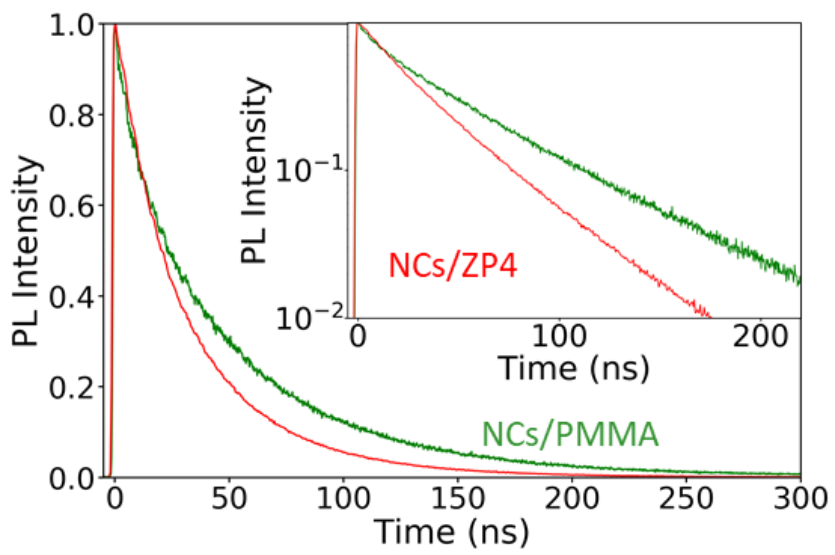

Figure S15: PL decay curves measured in ambient conditions with $405 \mathrm{~nm}$ excitation for $\mathrm{CsPbI}_{3} \mathrm{NCs}$ in $\mathrm{ZP} 4$ and PMMA composites shown in Figure 3 of the main text, normalized at $t=0$. Inset: PL decay curves plotted on a log scale. 
Table S2. Fitting parameters from biexponential and monoexponential fits to the PL decay data shown in Figure S15.

\begin{tabular}{|c|c|c|}
\hline parameter & PMMA & ZP4 \\
\hline$\tau_{1}$ & $12.5 \mathrm{~ns}$ & $32.3 \mathrm{~ns}$ \\
$\% \tau_{1}$ & $29.8 \%$ & $100 \%$ \\
$\tau_{2}$ & $58.8 \mathrm{~ns}$ & \\
$\% \tau_{2}$ & $70.1 \%$ & \\
$\tau_{\text {avg }}$ & $55.0 \mathrm{~ns}$ & $32.3 \mathrm{~ns}$ \\
\hline
\end{tabular}
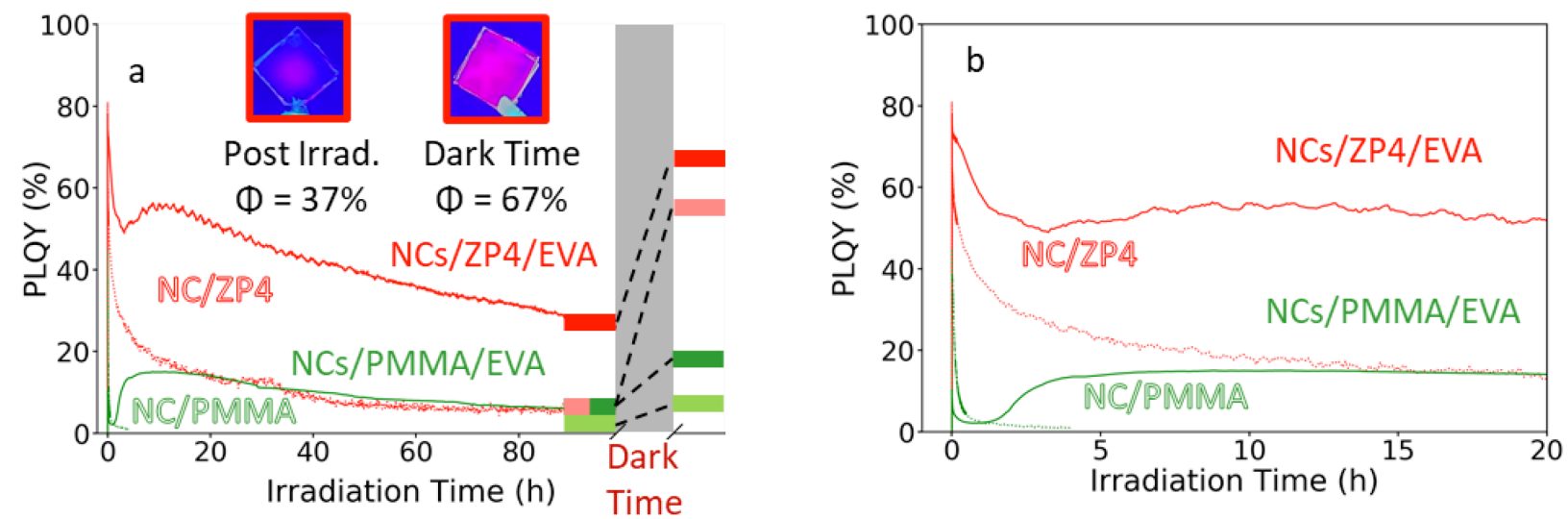

Figure S16. (a) PLQY as a function of $450 \mathrm{~nm}$, ambient irradiation time for $\mathrm{CsPbI}_{3}$ NC/ZP4 and NC/PMMA composites with and without EVA encapsulation. The PLQY values measured before and after dark storage are indicated as horizontal bars. Inset: Photographs of an EVA-laminated $\mathrm{CsPbI}_{3} \mathrm{NC} / \mathrm{ZP} 4$ composite taken immediately after irradiation and after 2 months of dark storage under ambient conditions. PLQYs are provided below each image. (b) PLQY as a function of $450 \mathrm{~nm}$, ambient irradiation time for NC/ZP4 and NC/PMMA composites with and without EVA, plotted over the first 20 hours of irradiation.

Figure $\mathrm{S} 16 \mathrm{a}, \mathrm{b}$ show that the PLQY of the $\mathrm{CsPbI}_{3} \mathrm{NC} / \mathrm{ZP} 4$ composite is better preserved under irradiation than the PLQY of the NC/PMMA composite. Furthermore, the PLQY of the $\mathrm{NC} / \mathrm{ZP} 4$ composite recovers to $67 \%$ of its original value after 14 days of dark storage, whereas that of the NC/PMMA sample only recovers to $16 \%$ of its original value after dark storage. To understand the role of air in the $\mathrm{NC}$ photodegradation, we also laminated $\mathrm{CsPbI}_{3} \mathrm{NC} / \mathrm{ZP} 4$ and $\mathrm{CsPb}_{3} \mathrm{NC} / \mathrm{PMMA}$ composites with EVA, and $\mathrm{PL}$ results from these laminated composites under irradiation are included in Figure S16a,b. The PLQY of the NC/ZP4 composite remains high $(\sim 80 \%)$ immediately following lamination, whereas that of the NC/PMMA composite drops to only $\sim 10 \%$ after lamination. Under illumination, the PLQYs of both composites are better preserved when encapsulated in EVA, suggesting that exposure to a combination of air and light is responsible for the decreases in PLQY observed here. The photographs in Figure S16a show that the $\mathrm{NCs}$ at the edges of the EVA-laminated $\mathrm{CsPbI}_{3} \mathrm{NC} / \mathrm{ZP} 4$ composite degrade first, confirming that water and/or oxygen diffuses inward from the edges of the laminated composite to degrade the NCs while they are irradiated. A PLQY of 37\% was measured 1 day after 
irradiation. Interestingly, we found that even these edge NCs recover their luminescence, returning the PLQY of the entire $\mathrm{CsPbI}_{3} \mathrm{NC} / \mathrm{ZP} 4 / \mathrm{EVA}$ composite to $52 \%$ after 10 days of dark storage and $67 \%$ after 50 days of dark storage. These results suggest that NC PLQY recovery requires both dark storage time and air exposure. The $\mathrm{CsPbI}_{3}$ NC/PMMA/EVA composite recovers some PLQY within the first few hours of irradiation and recovers its PLQY after dark storage, but its absolute PLQY remains low.
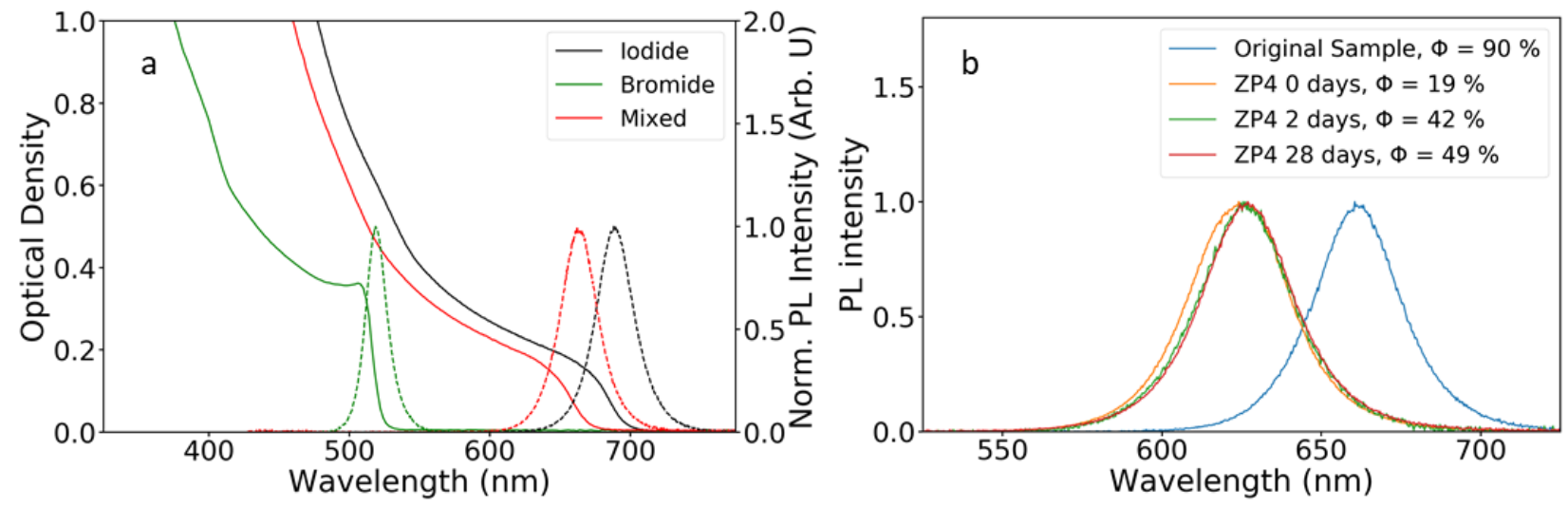

Figure S17. (a) Absorption and PL spectra of $\mathrm{CsPbI}_{3}$ (black) and $\mathrm{CsPbBr}_{3}$ (green) $\mathrm{NCs}$ in hexanes solutions. Aliquots of these solutions were combined to yield mixed $\mathrm{CsPb}\left(\mathrm{Br}_{1-x} \mathrm{I}_{x}\right)_{3} \mathrm{NCs}$ (red). (b) PL spectra of the as-synthesized $\mathrm{CsPb}\left(\mathrm{Br}_{1-x} \mathrm{I}_{x}\right)_{3} \mathrm{NCs}$ and the same NCs in a ZP4 composite. Transfer into ZP4 was accompanied by a $50 \mathrm{~nm}$ blue shift, but the PLQY remained high after nearly a month of dark storage. 
Table S3. Summary of publications that report successful stabilization of $\mathrm{CsPbI}_{3}$ or $\mathrm{CsPb}\left(\mathrm{Br}_{1-x} \mathrm{I}_{x}\right)_{3} \mathrm{NCs}$ in solid composites. The solid-state PLQYs of these composites are also provided.

\begin{tabular}{|c|c|c|c|}
\hline ref. & Matrix description & $\begin{array}{c}\text { Used for } \\
\mathrm{CsPbI}_{3} \mathrm{NCs} \\
(x=1) \\
\end{array}$ & PLQY (\%) \\
\hline $\begin{array}{l}\text { Di Stasio et } \\
\text { al. }^{10} \\
\end{array}$ & $\begin{array}{l}\mathrm{PbBr}_{2} \text { treated, anion } \\
\text { exchanged film }\end{array}$ & yes & 31 \\
\hline Li et al. ${ }^{11}$ & silica microsphere bound & no & 35 \\
\hline Cai et al. ${ }^{12}$ & poly(2-ethyl-2-oxazoline) & no & 45 \\
\hline Xuan et al. ${ }^{13}$ & \begin{tabular}{|c|}
$\begin{array}{c}\text { porous organic polymer } \\
\text { network }\end{array}$ \\
\end{tabular} & yes & 37 \\
\hline Wei et al. ${ }^{14}$ & epoxy resin composite & yes & 17 \\
\hline Zhang et al. ${ }^{15}$ & $\begin{array}{c}\text { polymer microsphere } \\
\text { precipitation }\end{array}$ & no & 40 \\
\hline Chen et al. ${ }^{16}$ & PMMA & yes & 48 \\
\hline Zhang et al. ${ }^{17}$ & $\mathrm{CsPbI}_{3}: \mathrm{CsPbCl}_{3} \mathrm{NC}_{\text {glass }}$ & yes & 45 \\
\hline Wu et al. ${ }^{18}$ & $\begin{array}{c}\text { Triphenyl phosphine } \\
\text { treated film }\end{array}$ & yes & 99 \\
\hline
\end{tabular}

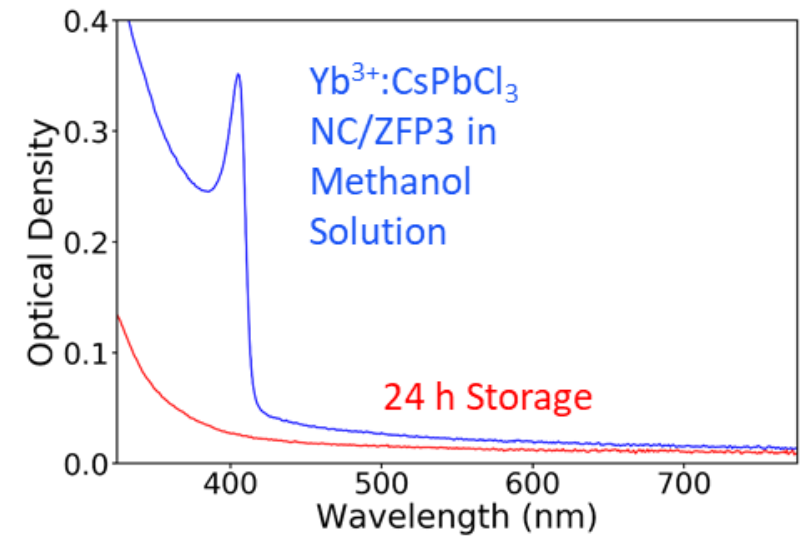

Figure S18. Absorption spectra of $\mathrm{Yb}^{3+}: \mathrm{CsPCl}_{3} \mathrm{NC} / \mathrm{ZFP} 3$ composites suspended in methanol initially and after 24 hours of dark ambient storage. The NCs degraded in the presence of methanol. No comparable degradation is observed when butyl acetate is used as the solvent instead of methanol.

\section{References}

1. Nenon, D. P.; Pressler, K.; Kang, J.; Koscher, B. A.; Olshansky, J. H.; Osowiecki, W. T.; Koc, M. A.; Wang, L.-W.; Alivisatos, A. P. Design Principles for Trap-Free CsPbX Nanocrystals: Enumerating and Eliminating Surface Halide Vacancies with Softer Lewis Bases. J. Am. Chem. Soc. 2018, 140, 17760-17772. 
2. Milstein, T. J.; Kroupa, D. M.; Gamelin, D. R. Picosecond Quantum Cutting Generates Photoluminescence Quantum Yields Over 100\% in Ytterbium-Doped $\mathrm{CsPbCl}_{3} \mathrm{Nanocrystals}$ Nano Lett. 2018, 18, 3792-3799.

3. Makarov, N. S.; Guo, S.; Isaienko, O.; Liu, W.; Robel, I.; Klimov, V. I. Spectral and Dynamical Properties of Single Excitons, Biexcitons, and Trions in Cesium-Lead-Halide Perovskite Quantum Dots. Nano Lett. 2016, 16, 2349-2362.

4. Utzat, H.; Shulenberger, K. E.; Achorn, O. B.; Nasilowski, M.; Sinclair, T. S.; Bawendi, M. G. Probing Linewidths and Biexciton Quantum Yields of Single Cesium Lead Halide Nanocrystals in Solution. Nano Lett. 2017, 17, 6838-6846.

5. Sillen, A.; Engelborghs, Y. The Correct Use of "Average" Fluorescence Parameters. Photochem. 1998, 67, 475-486.

6. Chirvony, V. S.; González-Carrero, S.; Suárez, I.; Galian, R. E.; Sessolo, M.; Bolink, H. J.; Martínez-Pastor, J. P.; Pérez-Prieto, J. Delayed Luminescence in Lead Halide Perovskite Nanocrystals. J. Phys. Chem. C 2017, 121, 13381-13390.

7. Rabouw, F. T.; van der Bok, J. C.; Spinicelli, P.; Mahler, B.; Nasilowski, M.; Pedetti, S.; Dubertret, B.; Vanmaekelbergh, D. Temporary Charge Carrier Separation Dominates the Photoluminescence Decay Dynamics of Colloidal CdSe Nanoplatelets. Nano Lett. 2016, 16, 2047-2053.

8. Marchioro, A.; Whitham, P. J.; Knowles, K. E.; Kilburn, T. B.; Reid, P. J.; Gamelin, D. R. Tunneling in the Delayed Luminescence of Colloidal CdSe, $\mathrm{Cu}^{+}$-Doped $\mathrm{CdSe}$, and $\mathrm{CuInS}_{2}$ Semiconductor Nanocrystals and Relationship to Blinking. J. Phys. Chem. C 2016, 120, 27040-27049.

9. Rabouw, F. T.; Kamp, M.; van Dijk-Moes, R. J. A.; Gamelin, D. R.; Koenderink, A. F.; Meijerink, A.; Vanmaekelbergh, D. Delayed Exciton Emission and Its Relation to Blinking in CdSe Quantum Dots. Nano Lett. 2015, 15, 7718-7725.

10. Di Stasio, F.; Christodoulou, S.; Huo, N.; Konstantatos, G. Near-Unity Photoluminescence Quantum Yield in $\mathrm{CsPbBr}_{3}$ Nanocrystal Solid-State Films via Postsynthesis Treatment with Lead Bromide. Chem. Mater. 2017, 29, 7663-7667.

11. Li, X.; Wang, Y.; Sun, H.; Zeng, H. Amino-Mediated Anchoring Perovskite Quantum Dots for Stable and Low-Threshold Random Lasing. Adv. Mater. 2017, 29, 1701185.

12. Cai, W.; Chen, Z.; Li, Z.; Yan, L.; Zhang, D.; Liu, L.; Xu, Q.-h.; Ma, Y.; Huang, F.; Yip, H.L., et al. Polymer-Assisted in situ Growth of All-Inorganic Perovskite Nanocrystal Film for Efficient and Stable Pure-Red Light-Emitting Devices. ACS Appl. Mater. Interfaces 2018, $10,42564-42572$.

13. Xuan, T.; Huang, J.; Liu, H.; Lou, S.; Cao, L.; Gan, W.; Liu, R.-S.; Wang, J. SuperHydrophobic Cesium Lead Halide Perovskite Quantum Dot-Polymer Composites with High Stability and Luminescent Efficiency for Wide Color Gamut White Light-Emitting Diodes. Chem. Mater. 2019, 31, 1042-1047.

14. Wei, S.; Zhu, H.; Zhang, J.; Wang, L.; An, M.; Wang, Y.; Zhang, X.; Liu, Y. Luminescent Perovskite Nanocrystal-Epoxy Resin Composite with High Stability Against Water and Air. J. Alloys Compd. 2019, 789, 209-214.

15. Zhang, C.; He, Z.; Chen, H.; Zhou, L.; Tan, G.; Wu, S.-T.; Dong, Y. Light Diffusing, DownConverting Perovskite-on-Polymer Microspheres. J. Mater. Chem. C 2019, 7, 6527-6533.

16. Chen, C.; Li, D.; Wu, Y.; Chen, C.; Zhu, Z.-G.; Shih, W. Y.; Shih, W.-H. Flexible Inorganic $\mathrm{CsPbI}_{3}$ Perovskite Nanocrystal-PMMA Composite Films with Enhanced Stability in Air and Water for White Light-Emitting Diodes. Nanotechnology 2020, 31, 225602. 
17. Zhang, Z.; Shen, L.; Zhao, Y.; Zhang, Y.; Yang, H.; Xiang, W.; Liang, X.; Chen, G.; Yu, H. Coexisting $\mathrm{CsPbCl}_{3}: \mathrm{CsPbI}_{3}$ Perovskite Nanocrystal Glasses with High Luminescence and Stability. Chem. Eng. J. 2020, 385, 123415.

18. Wu, J.; Tong, J.; Gao, Y.; Wang, A.; Zhang, T.; Tan, H.; Nie, S.; Deng, Z. Efficient and Stable Thin-Film Luminescent Solar Concentrators Enabled by Near-Infrared Emission Perovskite Nanocrystals. Angew. Chem. Int. Ed. 2020, 59, 7738-7742. 